Au carrefour des cultures : Marie-Aimée Steck-Guichelin

Pour Bernard Steck

Dans le numéro XIX, du 1er germinal an 9 (22 mars 1801), du Mercure de France, deux textes poétiques se suivent. Le «Fragment d'une Epître à l'Obscurité, par une jeune personne de dix-sept ans » précède une «Elégie dans le goût ancien par feu André Chénier ». Le second morceau est devenu célèbre sous le titre de «La Jeune Tarentine ». Lectrice assidue, Isabelle de Charrière, qui se dit «aussi approuvante et admirante [sic] que j'ai coutume d'être le contraire», dans une lettre du 1er avril 1801 à Benjamin Constant, commente ces publications : «Les vers que je trouve dans ces deux derniers Mercures $\mathrm{N}^{\circ}$ s $18 \& 19$ sont charmants surtout les fragments d'une épître à l'obscurité - Non, ils ne sont pas plus jolis que l'élégie du pauvre André Chénier n'est touchante, simple, antique. » Avec les «Réflexions sur les femmes, par une femme», qui constituent une réaction à De la littérature de Germaine de Staël et sont peut-être de Pauline de Meulan, ${ }^{1}$ ces différentes publications dans la presse constituent, pour la dame du Pontet, des indices d'un retour bienvenu à une forme d'équilibre : «Je crois en vérité qu'on commence à changer de style, qu'on revient à celui du bon sens. »²

Le Mercure de France ne livre que 80 vers des 156 alexandrins à rimes plates que compte l'« Epître à l'Obscurité ». Celle-ci n'était pas inédite, à la différence de l'élégie de Chénier - elle avait même été lue au Lycée en l'an $7-,{ }^{3}$ mais elle se retrouve à la place d'honneur en ouverture d'un des périodiques les plus lus du temps. Attribuée à « une jeune personne de dix-sept ans », elle est l'œuvre d'une Versaillaise d'origine, née Marie-Aimée Guichelin, épouse de celui qui fut premier secrétaire de la république helvétique, Jean Rodolphe Steck. La périphrase qui la désigne, sans révéler son nom, est typique de son désir de rester dans l'ombre, du moins à l'âge adulte. Elle est au carrefour de deux tendances paradoxales : celle qui veut voir les femmes et les hommes acquérir une éducation comparable et celle qui restreint l'univers féminin à la sphère intime. Elle est également au carrefour de plusieurs mondes car elle fera, toute sa vie, œuvre de passeur culturel. Je souhaite esquisser ici quelques grandes lignes la concernant. Revenons pour commencer sur les débuts d'un auteur talentueux dont le nom ne figure que rarement dans les annales.

\title{
Une jeunesse littéraire
}

Née à Versailles le 30 janvier 1776, Aimée Guichelin (la future Mme Steck) a pour parents un maitre menuisier et une ouvrière en linges qui meurt tôt - le 20 octobre 1787. ${ }^{4}$ Son milieu est celui des artisans urbains dans lequel l'alphabétisation est devenue la norme, même pour les femmes. Les professions exercées par ses proches sont essentiellement manuelles, mais l'entourage des Guichelin comprend aussi un clerc de notaire ou un garçon libraire entre autres, 0 signataires des actes concernant la famille dans les archives paroissiales. ${ }^{5}$ Nous ne savons pas grand chose de l'éducation initiale de la petite fille, qui a plusieurs frères et sœurs. E. Michaud, "professeur à l'université de Berne », qui publie en 1885 une petite brochure consacrée à Mme Steck et ses poésies,

\footnotetext{
1 Voir Silvia Lorusso : «Sophie Cottin face à Germaine de Staël », in : Les Femmes en mouvement. L'Univers sentimental et intellectuel des romancières du début du XIXe siècle, Fabienne Bercegol et Cornelia Klettke éd. (Berlin, 2016$) 76$.

2 «Lettre $2280 »$ dans Isabelle de Charrière/Belle de Zuylen : Correspondance t. VI (1800-1805), texte établi et annoté par Jean-Daniel Candaux, Simone et Pierre H. Dubois, avec la collaboration de Michel Gilot (Amsterdam, 1984, 247-248). L'orthographe de toutes les citations a été modernisée.

${ }^{3}$ Lettre de Pougens, le 20 vendémiaire an 7 . FA Steck. Sur le poème et sa diffusion, je me permets de renvoyer à mon article "Les réseaux franco-helvétiques de Mme Steck-Guichelin », in : Les écrivains suisses alémaniques et la culture francophone au 18 siècle, éd. Michèle Crogiez Labarthe, Sandrine Battistini et Karl Kürtös (Genève, 2008) 371-380.

${ }^{4}$ Comme j'ai eu l'occasion de le relever, son acte de baptême lui donne les prénoms de « Marie-Anne ». Peut-être est-ce une erreur de transcription du curé. Elle est en tout cas appelée Aimée ou Marie-Aimée et c'est ainsi qu'elle signe (Marie Aimée Guichelin est très lisible sur son certificat de mariage).

${ }^{5}$ Sur les origines familiales d'Aimée Guichelin et ses relations avec le poète Le Brun, je me permets de signaler mon article «Une Versaillaise oubliée : Aimée Steck-Guichelin, la Thémire de Le Brun », Cahiers Roucher - André Chénier n²3 (2004) 143-169.
} 
y évoque un passage de l'enfant dans un couvent à Maisons, près de Paris, et sa composition, à l'âge de huit ans, d'un quatrain pour mettre au bas du portrait de Henri IV :

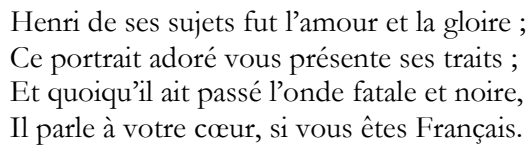

Aucune source extérieure ne vient confirmer ces affirmations, fondées a priori sur une tradition familiale - le biographe a fréquenté Ernestine Wyss, née Steck, la fille d'Aimée Steck-Guichelin, ainsi que le professeur de théologie Rudolf Steck, son petit-fils -, si ce n'est la présence, dans un poème plus tardif, d'une allusion à Maisons, « lieu si cher à ma jeunesse ». Michaud ajoute que la fillette fut ensuite produite dans les salons parisiens par l'ecclésiastique qui était son professeur à Maisons. ${ }^{6} \mathrm{Si}$ nous en sommes, pour l'instant, réduits à des hypothèses sur les jeunes années d'Aimée Guichelin, des documents viennent confirmer son goût précoce pour la poésie : son nom commence à figurer très tôt dans les pages de la presse périodique. Prenons l'exemple du Journal général de France du samedi 3 novembre 1787 : le publiciste y livre des détails à propos du petit prodige grâce à une lettre reçue avec sa première publication, "Vers sur la mort de Léopold, Duc de Brunswick; par la Demoiselle Guichelin, âgée de onze ans, fille du sieur Guichelin, Me Menuisier à Versailles, A Versailles, de l'Imprimerie de Pierres, premier Imprimeur ordinaire du Roi, 1787, 8 pag. In- $8^{\circ} »$. Voici le début de l'article :

\begin{abstract}
L’envoi qui m’a été fait de cette Pièce de vers a été accompagné d'une Lettre de M. Petit, Professeur au Collège d'Orléans, conçue en ces termes. "L'âge tendre de cette enfant (Mlle Guichelin) la tournure de certains vers, feront peut-être douter qu'elle soit l'Auteur de ce petit ouvrage, malgré les défauts qu'il renferme. Je prends la liberté de vous écrire, pour vous assurer que cette jeune Demoiselle a composé elle-même cette Pièce, qu'elle en a dirigé le plan, après s'être remplie de son sujet sur la narration que fit, alors de cet événement, M. le Rédacteur du Mercure, à l'article Francfort. Des personnes respectables et dignes de foi, qui, comme moi, l'ont vue travailler, vous attesteront, s'il le faut, qu'elle n'a été aidée de personne. Les différentes Pièces qu'elle a composées, ne sont, comme celle-ci, que le fruit de la lecture, pour laquelle elle a eu, dès l'âge de quatre ans et demi, un goût décidé. A huit ans, pénétrée du sujet d'une tragédie qu'elle venait de lire, il lui prit fantaisie de composer, en prose, quatre ou cinq scènes, où des personnes instruites aperçurent quelques étincelles de génie. Son père, profitant des avis qu'on lui donna de pourvoir à son éducation, se forma une Bibliothèque assez bien composée pour un homme de son état. Chacun s'empressa dès lors à lui fournir de bons Auteurs. C'est la lecture seule qui a développé son goût inné pour la versification, et qui l'a mise en état de faire beaucoup de Pièces fugitives bien meilleures que celle-ci, qui doit son impression à la célébrité que donna à ce sujet le Prix proposé à la plus belle Ode. Des Maitres instruits, étonnés de ses dispositions, lui donnent, depuis six mois, des leçons gratuites de latin (elle explique Virgile littéralement) et de dessin. L'impression de cet ouvrage étant faite au profit de l'Auteur, je vous prie, Monsieur de prévenir le Public en sa faveur. ${ }^{7}$
\end{abstract}

Plusieurs éléments sont dignes d'être relevés : ils placent la débutante dans un moule. Elle a un goût marqué pour la lecture depuis le départ. Elle a appris la versification toute seule. Elle étonne les maitres. Un paradigme analogue est utilisé quelques années plus tard par Victoire Babois pour évoquer ses propres dispositions poétiques : un penchant pour la littérature, une formation d'autodidacte d'abord, appuyée sur un programme de lecture sérieux par la suite et des conseils éclairés de professionnels. ${ }^{8}$ La lettre de Petit fait de l'enfant qu'il introduit une sorte de pupille de la république des lettres, qui reçoit des leçons et devrait toucher un modeste revenu de la vente de son opuscule. Notons au passage que, malgré son nom, le Collège d'Orléans est un établissement du quartier Notre-Dame de Versailles, la ville où la jeune Aimée Guichelin est née. Petit était le professeur de cinquième. ${ }^{9}$

Nous ne connaissons pas d'exemplaire du texte sur la mort de Léopold de Brunswick composé par la Versaillaise - peut-être s'en retrouvera-t-il un jour au détour d'un recueil factice. Il est impossible de savoir s'il a été envoyé au concours de l'Académie française auquel fait allusion le journaliste et qui visait à faire célébrer le défunt Léopold de Brunswick comme une figure hérö̈que de prince philosophe : les archives de l'Institut concernant les prix n'ont pas été conservés pour ces années-là. En revanche, grâce à la suite de l'article, nous

${ }^{6}$ E. Michaud, Mme Steck et ses poésies. 1776-1821 (La Chaux-de-Fonds, Imprimerie du National Suisse, 1885) 3-4.

7 Journal général de la France, n 132 , p. 526.

8 Voir « Victoire Babois (1760-1839)», Femmes poètes du XIXe siècle, une anthologie, sous la direction de Christine Planté (Lyon, 2010 [1998]) 83-84.

${ }^{9}$ Voir l'Almanach de Versailles, Année 1789 (Versailles et Paris, 1788) 281-2. 
pouvons en livrer 22 alexandrins, dûment introduits par le journaliste. Ils n’ont, je crois, jamais été reproduits - Michaud ne parait pas en avoir eu connaissance :

On imagine bien que je ne porterai pas le flambeau de la critique sur la production de cette enfant. Je lui dois au contraire des encouragements, et l'exhorter à cultiver un talent si précoce, et qui s'annonce d'une manière si singulière. Pour mettre le lecteur à portée d'en juger, je citerai quelques vers.

Brunswick, seul au milieu des communes alarmes,

Met dans sa fermeté son espoir et ses armes;

Ranimé, soutenu par son cœur bienfaisant,

Il redouble l'effort de son bras chancelant;

Il prodigue sa vie, il lutte contre l'onde ;

Le destin de Brunswick semble celui du monde ;

Et ses jours consacrés aux vertus, aux bienfaits,

Paraissent moins les siens que ceux de ses sujets.

Mais du sein de l'Oder une vague s'élance,

Et soudain vers le Prince en mugissant s'avance.

A cet aspect affreux, le cri de la douleur

Du péril qui l'attend devient l'avant-coureur ;

Tous les yeux sont fixés sur l'onde meurtrière :

Tout gémit... mais Brunswick a revu la lumière,

Leur Prince vit encore, et pour eux ranimé,

Il rend la confiance à leur cœur alarmé ;

Leurs craintes, leur effroi se calment à sa vue...

Mais déjà sans vigueur sa main est suspendue

Aux branches d'un arbuste, à de faibles rameaux ;

Ils se rompent... Brunswick retombe au sein des eaux ;

L'onde mugit, s'entr'ouvre, et le Prince succombe :

Elle est de ses vertus le théâtre et la tombe.

S'il faut parcourir les pages des journaux pour trouver la moindre syllabe de ce premier poème publié d'Aimée Guichelin, dont certains vers, comme l'affirme le Mercure de France, plus enthousiaste que l'Année littéraire, ${ }^{10}$ sont «très heureusement tournés [...], étonnent de la part d'une jeune personne de 10 ans et demi, et $[\ldots]$ annoncent un talent fait pour être encouragé $»,{ }^{11}$ sentiment que le lecteur moderne peut tout à fait partager, il faut bien chercher, par la suite, pour retrouver des mentions ou des citations de ses œuvres.

Le succès prématuré de l'auteur - rappelé dans un poème de Le Brun rédigé à l'occasion de son départ pour la Suisse ${ }^{12}$ - lui vaut d'être conviée aux festivités organisées à Beauvais à l'occasion de l'inauguration d'une importante statue équestre du Roi Louis XIV. ${ }^{13}$ Le 12 août 1788 ont lieu les exercices publics et la distribution des prix des écoliers de la ville. «Une jeune fille de douze ans, mademoiselle Guichelin, connue par une pièce de vers publiée en 1787 sur la mort de Léopold de Brunswick, avait été invitée à la séance. L'élève Leclercq, de Pont-Sainte-Maxence, obtint le premier prix, et mademoiselle Guichelin y joignit un exemplaire de son poème qu'elle offrit au vainqueur. Un autre prix ayant valu un instant après, une seconde couronne au même élève, celui-ci s'empressa d'en faire hommage à la jeune muse. ${ }^{14}$

J'ignore quelles ont été les autres occupations de l'enfant talentueuse. Je ne la retrouve pas dans les pages des journaux après sa célébration du prince philosophe, mort noyé pendant une crue de l'Oder. Il faut supposer qu'elle a continué de s'instruire et d'écrire. Michaud date de 1791 la composition de la pièce intitulée

${ }_{10}$ Année littéraire 1787, t. VIII, lettre I, p. 37 : «La Dlle. Guichelin, âgée de onze ans, fille du sieur Guichelin, maitre menuisier à Versailles, a répandu aussi de ses mains enfantines, quelques fleurs sur le tombeau de Léopold : sa pièce très faiblement écrite, mais dont la versification est douce et facile, doit être regardée comme un prodige par rapport à l'âge et au sexe de l'Auteur. »

11 Mercure de France, 1er décembre 1787, p. 45.

12 «Onze printemps de Thémire étaient l'âge ;/ Déjà Thémire avait l’art de rimer./ Onze printemps! elle savait charmer,/ Et le génie admirait son ouvrage !» (Voir Michaud, Mme Steck et ses poésies [voir note 6] 14).

${ }^{13}$ Selon l'article consacré à Marie-Anne [sic] Guichelin, dans les Recherches historiques et biographiques sur Versailles ;

Biographie sommaire des personnes illustres, célèbres, remarquables, etc., nées dans cette Ville, 2de édition, par M. Eckard (Versailles, Dufaure, 1836) : «L'inauguration qui eut lieu à Beauvais, en 1788, de la statue équestre de Louis XIV, lui inspira une pièce de vers qui fut très recherchée. » Nous n'avons pas de confirmation de cela.

14 C.-L. Doyen : Histoire de la ville de Beauvais, depuis le XIVe siècle (Beauvais, 1842) II, 222. 
«La Nuit ». Un extrait de ce texte, qu’il reproduit, servira en tout cas à montrer que la poésie d'Aimée Guichelin est marquée au coin de l'esthétique du temps avec cette allusion à Young, auteur des célèbres Night Thoughts :

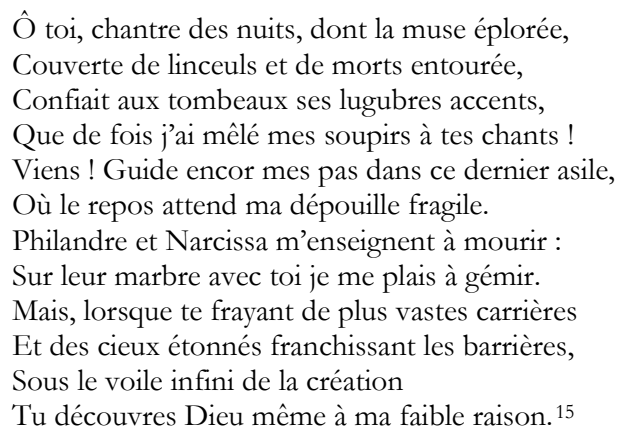

Selon Michaud, l'auteur n'aurait donc eu qu'une quinzaine d'années au moment de rédiger ces vers. Il n'étaye pas son affirmation par des documents précis.

\section{Des familles d'élection}

Aimée Guichelin aurait pu disparaitre complètement de l'horizon littéraire comme tant d'autres phénomènes éphémères de jeunes prodiges. Dans les faits, le personnage a été assez remarquable pour avoir laissé des traces dans des endroits parfois inattendus. Elle a eu la chance de rencontrer d'abord, par l'entremise des Silvestre, des Versaillais d'une lignée renommée d'artistes, les Brongniart, la famille de l'architecte de la Bourse de Paris, puis les Coquebert de Montbret. Grâce au «journal » adressé au père et au fils Brongniart, absents de la capitale, et dans la rédaction duquel, en 1793-1794, "la citoyenne Guichelin » joue un rôle important (elle était alors en quelque sorte l'institutrice de la dernière enfant de la famille, Emilie, et la secrétaire et dame de compagnie de sa mère), ${ }^{16}$ et à des mentions dans les papiers des Coquebert de Montbret, auprès desquels elle séjourne ensuite, jusqu'à son mariage en 1797 , nous parvenons à saisir des bribes du caractère de la jeune femme. Instruite et fine, elle suscite des élans de la part de ceux qui l'accueillent. Charlotte-Nicole Coquebert de Montbret, en particulier, restera, sa vie durant, fidèle à «son » Aimée, avec laquelle elle correspond après son départ pour la Suisse. Elle l'évoque pour la première fois dans un texte rédigé autour du 20 décembre 1794 :

Nous avons fait depuis un mois une acquisition bien intéressante pour notre intérieur. Nous avions fait il y a 2 ou 3 mois la connaissance plus particulière de la famille Brongniart et de la citoyenne Guichelin jeune personne très intéressante par ses connaissances et son caractère elle demeurait avec la citoyenne Brongniart et donnait des soins à l'éducation de son aimable fille. ${ }^{17}$

Mme Brongniart ne pouvait garder Aimée Guichelin auprès d'elle, a priori pour des raisons financières. Coquebert de Montbret a pensé que l'apprentissage de la langue allemande serait utile à la jeune femme : il a, avec sa famille, passé des années à Hambourg. Les cours particuliers se passent bien. Mlle Guichelin est intégrée à la famille Coquebert. Comme chez les Brongniart, elle est à la fois dame de compagnie, gouvernante et amie de la maison, au point que Mme Coquebert de Montbret l'appelle à l'occasion « ma fille Aimée » et que la jeune femme évoque son aînée comme « une seconde mère, une mère bien digne de ce nom ». Les Coquebert se montrent l'un et l'autre soucieux d'éduquer leurs enfants - et par extension d'éduquer Aimée - de manière à leur donner la possibilité de gagner leur vie. A une époque où les fortunes se font et, surtout, se défont du jour

\footnotetext{
15 Michaud, Mme Steck et ses poésies [voir note 6], p. 49.

${ }^{16}$ Sur la période, voir en particulier les documents reproduits par Louis de Launay dans « Une Vie de famille sous la Terreur », La Revue de France, 1935.

${ }^{17}$ Charlotte-Nicole Coquebert de Montbret, «Livre d'Ernest et de Cécile », in : Catriona Seth : La Fabrique de l'intime : Mémoires et journaux de femmes du XVIII' siècle (Paris, 2013) 894.
} 
au lendemain, avec les remous révolutionnaires, posséder une langue étrangère, c'est détenir un capital dont on ne peut être privé.

Les Brongniart, tout comme les Coquebert de Montbret, témoignent de l'importance accordée à l'éducation des deux sexes et de l'implication de parents dans la vie de leurs enfants. Les deux familles seront d'ailleurs alliées en 1800 lorsque Cécile (1782-1862), ${ }^{18}$ la fille des Coquebert de Montbret, épouse Alexandre Brongniart (1770-1847). Elles représentent l'une et l'autre cette frange de la société qui sans tenir le haut du pavé est bien introduite et fréquente différents milieux, parfois à la suite de contacts professionnels. Grâce à elles, Aimée Guichelin a croisé des personnalités de la fin de l'Ancien Régime. C’est ainsi que Louise Brayer de Saint-Léon l'évoque parmi les clients de la librairie de Charles de Pougens auquel la jeune femme, âgée alors d'environ dix-huit ans, fut présentée par Charlotte-Nicole Coquebert de Montbret, et pour lequel elle avait entamé la traduction d'un roman de Radcliffe en 1797, probablement sur la recommandation de l'éditeur qui devait flairer une opération commerciale intéressante. ${ }^{19}$ Et dans le salon de Magdalena Schweizer (1751-1814), née Hess, une proche de Füssli, Lavater et Pestalozzi, Aimée Guichelin a rencontré un patricien bernois, épris de philosophie et de liberté, qui avait étudié auprès de Fichte à Iéna. Leur première conversation aurait porté sur Kant. L'enthousiasme de Jean Rodolphe Steck (1772-1805) conquiert la Versaillaise et il la demande en mariage.

Pougens devait servir d'intermédiaire entre l'amoureux Helvète, alors secrétaire-substitut de la chancellerie d'État de Berne, et Maria Magdalena Steck-Jenner, l'austère mère bernoise de ce dernier, qui ne paraissait pas prête à accorder son consentement à l'union de son fils avec une intellectuelle sans fortune, étrangère de surcroît ${ }^{20}$. Les difficultés furent aplanies par des échanges de courriers, des recommandations et la requête directe du jeune amoureux à sa mère. Le couple fut uni le 30 septembre 1797 par le pasteur Gambs. Une lettre d'Aimée Guichelin à sa belle-mère, avant même d'arriver en Suisse, et qui est conservée dans les archives familiales, la montre comme prête à faire tous les efforts pour s'adapter : "Combien j’ai été touchée de la promesse que vous me faites de voir en moi la fille que vous avez perdue et de me rendre la mère dont j'ai été privée si jeune !» Elle ajoute une description d'elle-même : «Je serais bien fâchée qu'on s'attendît à voir ce qu'on entend ordinairement par une Française, c'est-à-dire de l'esprit, de la vivacité, des grâces, des talents agréables ; ce ne sera rien de tout cela ; mais une bonne et simple créature tout uniment, et rien autre chose. »

Les nouveaux mariés ont quitté Paris pour la Suisse où Suard les a rencontrés en octobre de la même année. Il évoquait alors dans une lettre à Meister Stebbel et Stapfer avant de les dire « liés l'un et l'autre à une jeune Française, mariée à M. Steck, et plein[e] de candeur, d'esprit et de talent». Il qualifiait l'époux de philosophe, partisan de Kant, préférant la jeunesse, l'esprit et la vertu à l'argent, bien que bourgeois de Berne. Il ajoutait : «Sa famille, qui n'est pas tout à fait aussi philosophe, a appris ce mariage avec un grand scandale. La jeune mariée est arrivée il n’y a pas un mois à Berne, effrayée des préventions qu'elle avait à vaincre ; au bout de huit jours elle a tourné toutes les têtes, mais par sa simplicité et sa douceur caressante plus que par son esprit et ses jolis vers. » ${ }^{21}$ Les témoignages concordent. Pierre Ochs rencontre le couple peu de temps après à Argovie - Jean Rodolphe Steck est alors secrétaire général du Directoire helvétique - et évalue ainsi l'épouse : «C’est une Parisienne à laquelle le citoyen Coquebert, membre de la commission pour les poids et mesures à Paris, prend un fort vif intérêt. Cette femme est poète, bel esprit et très fine. Elle écrit supérieurement bien. » ${ }^{22} \mathrm{Il}$ la

\footnotetext{
${ }_{18}$ Cécile Coquebert de Montbret : Journal (1799-1801), présentation et notes de Bernard et Pauline Poujeaux (Paris, 2007).

19 « Vos vers [à l'obscurité] ont été imprimés dans la décade avec les lettres initiales de votre nom seulement [...] mais disons un mot des mystères d’Udolphe. Le libraire Lepetit crie comme un enragé, quand aurez-vous fini ? ne pourriez-vous pas lui envoyer un peu de copie pour lui faire prendre patience ? » (Bibliothèque de la Bourgeoisie de Berne [BBB] FA Steck, lettre de Pougens, 7 nivôse an 6/27 décembre 1797). Le projet semble être tombé à l'eau. - Toutes les lettres à la famille Steck, sauf indication contraire, sont conservé dans le fonds FA Steck de la BBB.

${ }^{20}$ Voir les Mémoires et souvenirs de Charles de Pougens, commencés par lui et continués par Louise B. de Saint-Léon (Paris, 1834) $197-200$.

${ }^{21}$ Fonds Reinhart, Winthertur, 301-21, à Greng, le 18 octobre [1797].

22 Jean-René Suratteau : «Six lettres inédites de Pierre Ochs », Basler Zeitschrift für Geschichte und Altertumskunde (1967)

214. - Je corrige l'orthographe du patronyme Coquebert.
} 
juge « déjà bernoise dans l'âme ». Réformiste, Jean Rodolphe Steck sera limogé le 16 juin sur les instances du commissaire Rapinat. Le ménage s'installe alors à Moos-Seedorf. Jean-Rodolphe Steck meurt jeune, poitrinaire, en 1805, un an après avoir été fait membre de la cour d'appel du canton de Berne. Sa veuve resta avec trois fils et une fille.

Aimée Steck-Guichelin a entretenu une importante correspondance avec ses proches parisiens. En témoignent les 246 lettres qui lui ont été envoyées entre l’an V et 1821, essentiellement par Charlotte-Nicole Coquebert de Montbret, et le séjour que firent les amis français à Berne en 1817. Malheureusement la correspondance active de la jeune femme n'a pas survécu à notre connaissance. Ce goût pour les échanges et les lettres, explique peut-être son choix de traduire deux volumes de lettres de contemporains.

\section{Traduire}

Le projet de livrer une version française de Radcliffe, encouragé par Pougens, a été abandonné. Le mariage d'un côté, la publication de traductions de tiers de l'autre ont eu raison de la bonne volonté d'Aimée Guichelin. Elle n'abandonna néanmoins pas la pratique même si, de son propre aveu, elle était plus souvent tournée vers son intérieur et l'éducation de ses enfants que vers des activités potentiellement lucratives comme la traduction. Elle parait avoir consacré les revenus que lui offraient ces travaux à l'entretien des Guichelin restés en France.

Aimée Steck entreprend deux types de traduction : d'un côté des articles ou extraits destinés à la presse périodique, de l'autre des projets d'envergure. Sa première expérience dans le domaine remonte à son séjour parisien. Nous savons que Coquebert de Montbret a confié à sa protégée un texte destiné au Journal des Mines. $\mathrm{La}$ section intitulée "Extraits d'ouvrages étrangers» du $\mathrm{n}^{\circ} \mathrm{XV}$ annonce ainsi le : "Cinquième chapitre de la Géographie physique de Torbern Bergman ; traduit du suédois par la C [itoy]enne A. Guichelin ».23 Les époux Coquebert de Montbret sont certainement à l'origine de cette indication : la traductrice (qui a dû apprendre toute seule le suédois) veillera plus souvent à voiler son identité. Par la suite, de Suisse, Aimée Steck-Guichelin contribue à différents périodiques, et en particulier aux Archives littéraires dirigées par Suard, mais ses articles ne paraissent pas avoir été signés. On y trouve des allusions au détour d'échanges épistolaires ou des pages de son journal. Parmi les gens de lettres avec lesquels elle est en contact suivi, il y a Meister, rencontré par l'intermédiaire de Suard. Plusieurs billets d'elle à l'animateur de la Correspondance littéraire nous sont parvenus et il paraît avoir accordé un grand prix à son jugement.

Dans les premiers temps de son mariage, jeune mère de famille, Aimée Steck-Guichelin, dans une lettre à son mari, évoque le fait d'avoir accordé plus d'importance à son jeune fils Auguste qu'à la traduction de Gessner à laquelle elle se dit attachée. Elle a pourtant terminé sa version des lettres de famille de Gessner. En 1801, le Magasin encyclopédique célèbre, à l'article « Berne », cette nouvelle publication :

\footnotetext{
Il vient de paraitre ici un ouvrage intéressant pour les arts et la biographie, sous le titre de Correspondance de Salomon Gessner et de son fils Conrad (le peintre), publiée par le libraire Henry Gessner. Les lettres d'un tel père à son fils, sur son art et les moyens de s'y perfectionner, seraient du plus grand intérêt, sous le rapport des arts. Mais cet intérêt est encore augmenté par la gaieté et l'amabilité de l'auteur. Ce sera le plus beau monument érigé à Salomon Gessner. ${ }^{24}$
}

Rien ne vient indiquer qui est la traductrice et le titre de l'ouvrage est souvent cité de manière imprécise. Le même processus se répétera avec deux autres versions influentes de textes de grands contemporains pour lesquels l'intervention de la traductrice (et très souvent éditrice) est passée sous silence.

Une petite dizaine d'années plus tard, la traduction des Lettres de Jean de Muller à $V$. de Bonstetten et à Gleim, ${ }^{25}$ accompagnée d'une notice, est due à Aimée Steck-Guichelin. Cette correspondance (publiée sous le titre de Briefe eines jungen Gelehrten an seinen Freund) avait eu un grand succès en allemand. Avant de s'engager dans le travail, qui suppose, en termes de temps, un investissement considérable, la jeune femme consulte ses amis

${ }^{23}$ Numéro de frimaire [1796], p. 55. La traduction figure aux pages 55-79.

${ }^{24}$ Magasin encyclopédique, VII e année, t. I (1801), p. 266-267.

25 Zurich, 1810, 1 vol. in-18. 
parisiens, plus au fait qu'elle de ce qui est disponible ou souhaitable. La version que procure la Bernoise est saluée avec enthousiasme lors de la publication. Sismondi relève ainsi : "Le monde littéraire a été ici surtout occupé des lettres de Muller l'historien à notre ami Bonstetten. [...] Une dame vient de les traduire en français en en faisant un choix, et il serait difficile de trouver une lecture plus délicieuse. » ${ }^{26}$ Stapfer écrit plus tard : « Les Lettres de Muller à Bonstetten sont connues en France par une traduction qui en reproduit avec un extrême bonheur toute la verve et l'originalité ; on la doit à une dame bernoise, madame Steck, Française de naissance, qui a trouvé la célébrité malgré ses efforts pour cacher son nom. » ${ }^{27}$ Si Aimée Steck a ainsi permis aux Français de lire la correspondance familière d'intellectuels germanophones suisses, elle a élargi par la suite ses horizons.

Le troisième livre traduit par Aimée Steck-Guichelin, et qui était destiné à avoir une certaine influence, n'est en effet autre que l'Histoire de la littérature espagnole de Bouterwek, professeur à l'université de Goettingue. Le texte, en 2 volumes in- $8^{\circ}$, parait à Paris en 1812. Il s'agit d'une partie du panorama que l'Allemand, philosophe et historien, admirateur, mais aussi critique de Kant, a publié en plusieurs étapes entre 1801 et 1819 sous le titre Geschichte der neueren Poesie und Beredsamkeit (Histoire de la poésie et de la rhétorique modernes). La traductrice est également l'auteur de la préface de la version traduite. Comme pour les autres ouvrages allemands qu'elle a traduits, Aimée Steck-Guichelin ne fait pas apparaitre son nom. Quand elle en envoie un exemplaire à un proche, elle le revêt, en guise de dédicace, de la phrase suivante : «à Monsieur H. Meister de la part du Traducteur et de l'Editeur ». ${ }^{28}$ L'envoi n'est pas signé. Le nom de la traductrice (et on note la présence dans le rajout manuscrit du terme masculin) ne figure pas sur la page de titre. Certes, à l'époque, les traductions restent souvent anonymes, mais, on le voit, nous sommes face à un individu qui trouve son compte dans ce passage sous silence de son nom.

\section{Une muse discrète}

Ceux qui ont recherché les œuvres d'Aimée Steck-Guichelin n'ont pas eu une tâche simple. Le PostScriptum d'une missive de juin 1805 de la jeune femme à Meister indique ceci : «Puisque vous désirez lire la traduction du village abandonné, je vous envoie celle de M. Monvel, qui, je vous en assure, vaut beaucoup mieux que la mienne. » ${ }^{29}$ Comment ne pas comprendre les difficultés dont font état les bibliographes, aussi savants soient-ils! «On n’a pu découvrir si les poésies de cette dame ont été recueillies et imprimées, ni où parut la traduction qu'elle a donnée, en français, et qui est estimée, des Lettres d'un jeune savant à son ami, ou correspondance du célèbre histoiren Jean de Muller avec le savant Bonstetten. » ${ }^{30}$ écrit ainsi Eckard, l'auteur d'une biographie versaillaise parue sous la Restauration. Quérard, dans La France littéraire, cite les deux volumes des lettres et l'Histoire de la littérature espagnole, ainsi que la version française du Hameau abandonné de Goldsmith, avant d'ajouter: «Ces quatre traductions ont paru sous le voile de l’anonyme. Barbier, sous le $n^{\circ} 7528$, et à la table de son Dictionnaire, nomme, par erreur, le traducteur du dernier de ces quatre ouvrages Streck au lieu de Steck. » ${ }^{31}$ Il gratifie lui-même la femme de lettres d'une particule superflue la désignant comme "STECK (mademoiselle GUICHELIN, puis madame de)». Ajoutons qu'une autre de ses traductions, celle de l'Epître de Haller à Bodmer, a été publiée par erreur, quelques années après la mort de l'une et de l'autre, sous le nom d'une contemporaine bien plus célèbre qu'elle, Germaine de Staël, qu'elle a rencontrée à Berne. ${ }^{32}$

Dans son journal manuscrit, Aimée Steck-Guichelin rapporte que l'auteur de Delphine a beaucoup apprécié cette version au point de lui demander « de traduire quelques beaux morceaux des poètes allemands »,

\footnotetext{
${ }^{26}$ Lettres inédites de Sismondi (Paris, Lévy, 1863) 133.

${ }_{27}$ Philipp Albert Stapfer : Histoire et description des principales villes de l'Europe. Suisse. Berne (Paris, 1835) 83-4.

${ }^{28}$ Exemplaire conservé à la Fondation Reinhart à Winterthur.

${ }^{29}$ Fondation Reinhart, Winterthur, 1 298/1.

30 Article « Guichelin », Recherches historiques et biographiques sur Versailles ; Biographie sommaire des personnes illustres, célèbres, remarquables, etc., nées dans cette Ville, 2de édition, par M. Eckard (Versailles, 1836).

31 Article « Steck », Quérard, La France littéraire, t. IX (259-60).

32 Je me permets de renvoyer, pour les détails et sources concernant les échanges entre les deux femmes à mon article «Les rencontres entre Germaine de Staël et Marie-Aimée Steck-Guichelin », « Corinne », 200 ans après, Cahiers staëliens (2008) 4151.
} 
Bürger, Klopstock, Goethe... pour les adjoindre à un ouvrage qu'elle projette de faire imprimer - peut-être un état primitif de Corinne. Lors de leur conversation, qu'elle redoutait grandement, Aimée Steck est touchée par l'ouverture de son aînée qui lui indique être prête à faire toutes les démarches, à veiller à ce que les travaux soient rémunérés correctement et affirme même que personne n'a, pour le vers, le talent de son interlocutrice, avant d'ajouter: « je ne fais pas des vers comme vous. Je ne serai jamais une première personne en vers, au lieu que je puis me flatter d'en être une en prose; j'en reste donc à la prose. ».

S'il s'agit là encore d'un projet qui ne paraît pas avoir abouti, ce sont d'un côté sa transplantation dans une autre aire culturelle et sociale, et de l'autre son talent marqué pour les langues, qui ont donné à Aimée Guichelin un rôle d'intermédiaire aussi marqué. Michaud affirme : «Elle apprit et sut à peu près toutes les langues de l'Europe, sans excepter ni le latin, sur lequel elle était très forte, ni même le grec. » ${ }^{33} \mathrm{Il}$ évoque sa poésie sur la mort imitée du livre III de Lucrèce. Loin de la littérature, toujours selon Michaud : "Lors de l'annexion du Jura au canton de Berne, on eut recours à elle pour la traduction de plusieurs lois : le Code Napoléon ne l'effrayait aucunement, ni par ses termes juridiques, ni par sa sécheresse. » Ajoutons que deux des poèmes les plus longs d'Aimée Steck-Guichelin à être parvenus jusqu'à nous sont une traduction de l'allemand de Haller (son Epitre à Bodmer) et une version du Village abandonné de l'Irlandais Goldsmith (en 574 vers).

Aimée Guichelin est un passeur discret. Nous avons vu qu'elle ne se soucie pas de signer ses traductions. Elle est encore plus loin de vouloir voir publier ses propres vers, malgré l'insistance de ses proches. Lorsque parait pour la première fois son Epitre à l'Obscurité, ${ }^{34}$ Coquebert de Montbret est à l'origine de l'initiative. ${ }^{35}$ Quelques mots élogieux - ajoutés par l'économiste Jean-Baptiste Say ${ }^{36}$ - introduisent le morceau, œuvre "d'une très jeune personne », et qui " parait être le fruit d'un talent consommé ». Le départ de l'auteur en Suisse est décrit comme ayant " privé la France de beaucoup de vertus, de douceur, de connaissances et de talents. » Le poème a été repris, nous l'avons dit, par le Mercure de France, mais aussi par l'Almanach des muses et l'Almanach des dames. Il vaut à son auteur un quatrain élogieux de l'un des grands poètes du temps, Ponce-Denis Ecouchard-Le Brun, dit Le Brun-Pindare, et que lui adresse le 19 brumaire an 10 (10 novembre 1801), Charles Pougens :

Ta modestie enchanteresse

Brille d'un éclat mérité ;

Et tes vers à l'obscurité

N'iront jamais à leur adresse. ${ }^{37}$

Par la suite, les mêmes échos reviennent. Le 19 août 1804, Mme Coquebert de Montbret dit regretter que son amie s'étiole en Suisse étant donné ses talents. Son époux Coquebert aurait affirmé « c'est un meurtre qu'un esprit comme le vôtre ne s'emploie presque plus qu'aux détails du ménage. » Le 9 décembre 1805, il évoque une entreprise qui pourrait l'occuper de manière intéressante alors qu'elle pense disposer de deux ou trois heures par jour: "Il était question l'année dernière d'un Journal germanique et j'avais pensé à vous faire proposer d'y prendre part. Cette entreprise, provoquée dans l'origine par trois classes de l'Institut, a été mal vue ensuite ; il est peu probable qu'elle soit reprise ; si elle l'est, probablement j'en serai instruit. » Coquebert ne se remet décidément pas de l'absence de débouchés intellectuels de sa jeune protégée et tente de lui en procurer :

\footnotetext{
je ne puis m'accoutumer à l'idée de voir un si beau talent enfoui, tant de moyens anéantis par une modestie vraiment excessive. Quand je suis en train d'exagérer, j'appelle cela une ingratitude envers la Providence ; j'ai fort bien lu dans le temps tout ce que vous avez objecté à mes exhortations, mais je n'en ai pas été persuadé : j'y ai vu les sophismes d'une humilité trop grande qui se cache sous le masque de la paresse. Je ne crois point à votre paresse, je vous en avertis ; mais je crois à votre aversion pour la célébrité. Mais ne peut-on pas travailler utilement sans trop attirer les regards et l'envie, surtout lorsqu'on habite une petite ville et qu'on ne cesse pas d'être d'ailleurs tout ce qu'on doit être.
}

\footnotetext{
33 Michaud, Mme Steck et ses poésies [voir note 7] 4.

${ }^{34}$ Le poème paraît dans la Décade du 10 vendémiaire an VII, p. 35-36.

35 Charlotte-Nicole Coquebert de Montbret l'apprend à Aimée Steck-Guichelin dans une lettre du 30 novembre 1798.

${ }^{36}$ Lettre de la même du 25 novembre 1798.

${ }^{37}$ BNF NAF 9202 f 516.0
} 
Et son épouse, réagissant à ce qu'elle a su des conversations d'Aimée Steck avec une ainée fort célèbre, y va aussi de son commentaire : «Je suis bien aise que Mme de Staël vous ait engagée à travailler; il est si fâcheux de voir un beau talent comme le vôtre sans être exercé. »

\section{L'impossible bibliographie}

Au-delà des réticences sociales face à une femme qui écrit, Aimée Steck-Guichelin, comme le montrent ses cahiers, souffre de ce que les psychologues appellent un syndrome d'imposture. Elle a l'impression de ne pas être à la hauteur, de ne pas mériter les éloges qui lui sont décernés même par des proches ou des gens qu'elle admire. Lorsque Germaine de Staël, persuadée d'avoir en face d'elle une femme douée pour rendre en vers français la poésie étrangère lui demande de préparer des versions de grands textes rimés, elle convient de s'essayer aux traductions mais craint d'être paralysée par la requête. Elle assure que, quoi qu'il arrive, elle ne veut pas, si ses versions rimées sont publiées, voir citer son nom. L'échange entre celle que Rosalie de Constant appelle « la trop célèbre » ${ }^{38}$ et celle qui parait désirer l'anonymat est emblématique de son attitude. Aimée SteckGuichelin a tout fait pour cacher ses travaux. J'ignore donc la liste de ses œuvres complètes et n'ai pas réussi même à mettre la main sur toutes celles dont je connais l'existence. Il en reste des éléments dans les archives de sa famille. La modestie de la Bernoise d'adoption n'est pas feinte. Elle craint d'être exhibée au public - peutêtre a-t-elle été marquée par ses premières années de prodige poétique. Elle ne tient pas à diffuser ses sentiments à tout un chacun et préfère être le canal par lequel d'autres s'expriment. Elle affirme ainsi joliment, refusant la pression de proches qui l'enjoignent à publier ses vers, qu'elle aurait l'impression de se promener en sousvêtements (" en chemise» selon ses propres termes) dans la rue si on lisait sa poésie imprimée. Cela n’a pas empêché d'autres de souhaiter la voir paraitre. Stapfer affirme ainsi, avant de reproduire le poème de 1814 consacré par sa défunte amie à Napoléon agoni d'injures par la presse française : "Il serait à désirer qu'un choix des poésies et des pensées qu'elle a laissées en portefeuille vît le jour par les soins de sa famille. » ${ }^{39}$ Dans les faits, certains de ses vers ont été recueillis à la fin des Mélanges moraux, instructifs et amusans, ou Lectures pour la jeunesse, depuis l'âge de cinq à quinze ans, Lausanne, Imprimerie d'Emmanuel Vincent Fils, 1841. Elle y est désignée sous le nom incorrect de «Claire-Aimée St---k, née G----- ». Une note de l'éditeur à ce « Recueil de poésies, publiées par l'amitié » qui clôt le volume affirme : «L'auteur de ces charmantes poésies n'existe plus que dans le souvenir de ses amis ; ceux-ci n'auront pas de peine à le reconnaitre, malgré le voile qui couvre à moitié son nom, que des motifs particuliers empêchent de soulever en entier. » Michaud a livré à l'impression plusieurs textes avec le soutien de sa famille. Je dois à l'un de ses descendants d'avoir publié l'un de ses journaux intimes. ${ }^{40}$ Elle avait laissé son amie Charlotte-Nicole Coquebert de Montbret en recopier une partie. Dans un passage de cet écrit diaristique, elle évoque ses conversations avec Germaine de Staël en visite à Berne.

Elle [Mme de Staël] était intimement convaincue qu'on finissait toujours par obtenir la réputation qu'on mérite.

Fort bien, ai-je dit, mais à qui demander quelle est la réputation qu'on mérite ? Il faudrait le savoir d'avance avant de s'exposer, en cherchant la gloire, à ne rencontrer que le ridicule.

Elle n'a pas bien répondu à cette question. - Oh! m'a-t-elle dit on sent bien cela en soi-même.

Je suis donc condamnée, ai-je répondu, car je ne sens pas que j’ai un grand talent. Vous dites que je suis trop

modeste, mais croyez-vous que ce que vous appelez la modestie puisse se trouver en société avec le talent?

Je suis persuadée, me dit-elle.

Et moi, dis-je, j'en doute ; je crois que le talent a conscience de lui-même ; et comme vous l'avez dit, il se sent.

Deux visions se contredisent. Elles témoignent d'un plafond de verre ressenti par Aimée SteckGuichelin mais rejeté par Germaine de Staël - qui en paie à l'occasion le prix. Reconnue depuis l'enfance comme la fille de Necker, l'auteur des Réflexions sur le procès de la Reine est à l'aise avec sa propre identité - ou du moins sait qu'elle ne peut la rejeter, elle qui a été, selon ses propres dires, dans l'introduction à De l'influence des passions sur le bonheur des Individus et des nations en 1796, « condamnée à la célébrité ». Son interlocutrice, qui a changé de

\footnotetext{
38 Voir Catriona Seth, «Introduction », in : Mme de Staël, Euvres (Paris, 2017) xxvi.

${ }^{39}$ Stapfer, Histoire et description des principales villes de l'Europe. Suisse. Berne, [voir note 27] 80-1.

40 Aimée Steck-Guichelin, «Cahiers », in : C. Seth, La Fabrique de l’intime [voir note 17] 993-1049.
} 
milieu, plusieurs fois, et de pays, a moins de certitudes : elle est en marge par ses activités en tant que femme, issue d'un milieu populaire, et arrivée en Suisse grâce à son mariage. Elle se projette au travers des autres, ses enfants ou les traductions de tiers qui lui sont demandées ou qu'elle propose discrètement. Elle n'entend pas se doter d'une renommée propre et est blessée par l'affirmation indélicate de Staël selon laquelle son défunt époux aurait compté sur sa célébrité future : «j'ai dit que M. Steck à ce que j’espérais, m’avait épousée pour moi-même et non pour ma réputation. » Si le désaccord aurait pu menacer la conversation, c'est aussi l'occasion pour l'une et l'autre de tenter de se comprendre et de faire montre l'une envers l'autre d'ouverture et de générosité. Aimée Steck-Guichelin entend, même si elle se sous-estime, et elle avoue : « Je serais cependant assez curieuse de savoir quelle impression j’ai laissée à Mme de Staël. Son jugement ne peut pas m’être indifférent. »

Femme de lettres presque contre son gré, la discrète Versaillaise établie en Suisse ne cherche pas à être vue ou reconnue. Elle prête volontiers ses talents aux écrivains qu'elle traduit. Elle n'estime pas ses propres dons autant que le font ses proches. Ses contemporains saluent ses talents et ses connaissances. Il semble juste, comme elle a si souvent servi d'intermédiaire entre francophones et germanophones de terminer sur une appréciation en allemand, dans une correspondance privée : celle que formule Füssli dans une lettre à Jean de Müller. Aimée Steck-Guichelin y est décrite comme « obne Vermögen, aber von Charackter, Geist und ächter Kultur». ${ }^{41}$

\footnotetext{
${ }^{41}$ «Sans fortune, mais pleine de caractère, d'esprit et de culture véritable », d'après une lettre à Jean de Müller aimablement
} communiquée par Doris et Peter Walser (SH Mülleriana 61-12/87. Lettre du 12 juillet 1806). 